\title{
THE EFFECTIVENESS OF CARES DEMENTIA TRAINING MODULES ON DELIVERY OF PERSON CENTERED CARE INSIDE A MEMORY CARE UNIT: UTILIZING THE CARES OBSERVATIONAL TOOL
}

A Dissertation
presented to
the Faculty of the Graduate School
at the University of Missouri-Columbia
In Partial Fulfillment
of the Requirements for the Degree
Doctor of Nursing
be
ERIN CATTOOR

MAY 2018 
The undersigned, appointed by the dean of the Graduate School, have examined the dissertation entitled

THE EFFECTIVENESS OF CARES DEMENTIA TRAINING MODULES ON DELIVERY OF PERSON CENTERED CARE INSIDE A MEMORY CARE UNIT: UTILIZING THE CARES OBSERVATIONAL TOOL

presented by Erin Cattoor,

a candidate for the degree of doctor of nursing,

and hereby certify that, in their opinion, it is worthy of acceptance.

Marilyn J. Rantz

Gregory Alexander

Myra Aud

Colleen Galambos 


\section{ACKNOWLEDGEMENTS}

I would first like to dedicate my work to all of those that currently suffer with, care for, or will develop Alzheimer Disease or related dementias. It is my hope that one day in the near future; this research project will refer merely to a problem of the past. The population (and their families) referred to in my research truly represent my heroes, and inspire me personally and professionally on a daily basis.

I would be remiss if I did not also dedicate my work and my ability to pursue this endeavor for all of these years to many other individuals. My husband, Gil, has pushed me to keep going when I wanted to quit. We became a one-parent household at times, and he kept things running when I was not present mentally or physically. We have often referred to this process as a marathon, and I thank you for running it with me until the very end. My daughter, Avery. My sweet 9 year old who is wise beyond her years. You have truly been the inspiration for completing this degree. I know that I missed a lot of play time and sporting events in order to work. The greatest gift that I can give to you is this: Always finish what you start. No matter how long it takes you.

I also dedicate this work to my entire dissertation committee. I cannot thank you all enough for sticking with me throughout this very long process. Your individual contributions towards the field of gerontology and geriatrics is momentous. I am inspired and humbled by the work that you have done and continue to do, and hope to one-day make an impact on the geriatric population that will make you all proud. 


\section{TABLE OF CONTENTS}

ACKNOWLEDGEMENTS

ABSTRACT.

$\mathrm{V}$

\section{Chapter}

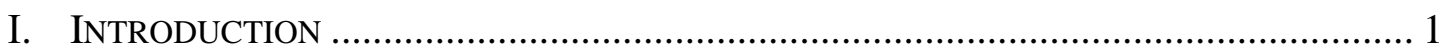

II. REVIEW OF RELATED LITERATURE ......................................................... 4

Alzheimer's disease and the Brain

Education of staff in LTC

Positive Physical and Psychosocial influence of online training modules on Person-Centered Care in LTC

Donabedian's Structure, Process and Outcomes (SPO) Framework

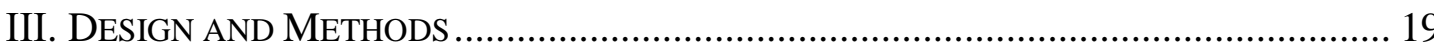

Research Question

Hypothesis

Sample

Procedures

Instrumentation

IV. RESULTS

Demographics

Hypothesis testing

Research Questions

V. DISCUSSION

Demographics

Alzheimer's disease and education of staff in MCUs

Hypothesis Findings 
Strengths/Limitations

Implications for Nursing

Implications for Research

Conclusion

BIBLIOGRAPHY

VITA 


\title{
THE EFFECTIVENESS OF CARES DEMENTIA TRAINING MODULES ON DELIVERY OF PERSON CENTERED CARE INSIDE A MEMORY CARE UNIT: UTILIZING THE CARES OBSERVATIONAL TOOL
}

\author{
Erin M. Cattoor
}

Dr. Marilyn J. Rantz, Dissertation Supervisor

\begin{abstract}
This study compared the efficacy of using online dementia training modules on both direct and non-direct care providers in long-term care settings and how this impacted their delivery of Person Centered Care (PCC), as well as their knowledge of caring for residents with a diagnosis of Alzheimer Disease (AD), dementia. Traditional educational opportunities for staff working specifically with demented residents inside Memory Care Units (MCU) were investigated, along with an alternative approach of training all staff (to include direct and non-direct care providers). The option of utilizing online dementia training modules for all staff was then evaluated by using an observational Person Centered Care tool, to see if education had made an impact on interactions between staff and the demented residents that they care for. This study utilized a single-group, repeated measures design to test a 10-week, standardized and computerized set of 10 interactive training modules in a 60-bed MCU . Fifty-one observations were made between MCU residents and staff and included in this study, employing a single-group pre-post-posttest design. The findings suggest that online dementia training modules may be beneficial for both knowledge and delivery of PCC to staff in MCUs who care for residents with a diagnosis of AD.
\end{abstract}




\section{Chapter I}

\section{Introduction}

A major goal of Healthy People 2020 is to maintain or enhance the quality of life for persons with dementia, including Alzheimer's disease (AD). In 2014, AD was the 6th leading cause of death among adults aged 18 years and older based on death certificate data (National Center for Health Statistics, 2015). Although estimates vary, analysis of data from the Chicago Health and Aging Project (CHAPS) and 2010 U.S. Census data suggests that the prevalence of dementia among adults aged 65 years and older in the U.S. in 2016 was $11 \%$, or 5.2 million people. Given these startling statistics, overall health and quality of life of those diagnosed with $\mathrm{AD}$ or related dementias must remain a top priority for those researching or working in long-term care (LTC).

Health care providers, consumers, and researchers are working diligently to develop and implement new models of care that fundamentally change the way we think about LTC services and how they are delivered (Crandall et al., 2007). Although no longer a novel idea, Person-centered care (PCC) is a key concept in the culture change movement, resonating as a global philosophy of care underpinning gerontological nursing (Nolan, 2001). PCC makes every effort to accomplish a better quality of life for elders and make LTC settings more desirable places to live and work (Rantz and Flesner, 2003). Despite the emergence of person-centered approaches and philosophies of care in LTC facilities, there is a paucity of data on the impact of these approaches on the well-being of residents, specifically on those with a diagnosis of AD or related dementias (Brownie \& Nancarrow, 2012). Standardized measurements of evaluating care provided to residents 
with dementia to evaluate delivery of PCC remains an area of needed research in clinical and scientific literature.

$\mathrm{AD}$ is a progressive brain disorder that damages and eventually destroys brain cells, leading to memory loss and changes in thinking and other brain functions. It usually develops slowly and gradually gets worse as brain function declines and brain cells eventually wither and die. Ultimately, AD is fatal, and currently, there is no cure. While there are different causes of dementia that are all associated with distinct symptoms and patterns of development, this study only focused on $\mathrm{AD}$, respectively. According to the Alzheimer's Association (2015), there are currently over 5 million people in Missouri living with a diagnosis of $\mathrm{AD}$, and as many as 16 million will have the disease by the year 2050. Additionally, one in three seniors who dies each year has AD or other dementias. AD denotes the sixth leading cause of death in the state of Missouri, representing the $9^{\text {th }}$ highest $\mathrm{AD}$ death rate in America. $\mathrm{AD}$ is the only cause of death among the top 10 in America that cannot be prevented, cured or even slowed (Alzheimer's Association, 2014).

The most common early symptom of AD is difficulty remembering newly learned information because $\mathrm{AD}$ changes typically begin in the part of the brain that affects learning (Alzheimer's Association, 2015). As AD advances through the brain it leads to increasingly severe symptoms, including disorientation; mood and behavior changes; deepening confusion about events, time and place; unfounded suspicions about family, friends and professional caregivers; serious memory loss and behavior changes; and difficulty speaking, swallowing and walking (Alzheimer's Association, 2015). Since AD is such a complex disease, encompassing a myriad of difficult to understand and treat 
symptoms, it is imperative that anyone caring for a person with the diagnosis of $\mathrm{AD}$ receive proper education and training to understand the disease and learn to better care for those inflicted. No research has been identified that describes potential correlations between improved PCC interactions to include both direct and non-direct care providers working exclusively within a Memory Care Unit, and exclusively with residents diagnosed with $\mathrm{AD}$ or related dementias.

To address this knowledge gap, this study measured dementia knowledge associated with utilization of CARES online dementia training modules with enhanced Person Centered Care (PCC) interactions among a sample of Midwestern U.S. direct and nondirect care providers in a MCU. The purpose of this study was to determine whether these educational interactions were also associated with successful duration of knowledge and ultimately, improved psychosocial relationships between staff and residents. Ultimately, this will translate into maintaining or enhancing the quality of life for residents with $\mathrm{AD}$ or related dementias, meeting a Healthy People 2020 objective (USDHHS, 2017). This study used a series of quantitative instruments to determine correlations between dementia knowledge (education) and PCC actions in direct and non-direct workers inside a MCU. Study subjects were all women (by chance), invited to participate in the study during their shifts, and participated through three different time points.

The aim of this study was to evaluate for perceived changes in PCC care after completing the CARES dementia training modules, using the CARES Observational Tool (COT) over a five-month period (January 2015-May 2015), as well as evaluate for changes in dementia care knowledge after completing the CARES dementia training modules, using the CARES Dementia Knowledge Test (DKT). 


\section{Chapter II}

\section{Review of Related Literature}

This chapter has four primary sections. The first section reviews Alzheimer's disease, as it relates to dementia, covering characteristics of $\mathrm{AD}$ to include cognitive deficits. The second section reviews research related to educational disparities related to direct and non-direct care workers in long-term care (LTC), and the benefit of online dementia training modules. The third section describes research related to the physical and psychosocial impact of online training modules on Person-Centered Care (PCC) for AD residents. The final section describes Donebedian's Structure, Process and Outcomes framework and how it guided this study.

\section{Alzheimer's disease and the Brain}

$\mathrm{AD}$ is the most common type of dementia, representing over $60 \%$ of all neurodegenerative-cognitive diagnoses. This chronic, neurodegenerative disease was first identified in 1901 by Alois Alzheimer, after studying the behaviors of a woman with short-term memory loss. Upon autopsy of his former patient's brain, a number of pathological conditions were identified, which continue to be the hallmark microscopic identifiers for a formal diagnosis of $\mathrm{AD}$. $\mathrm{AD}$ is a progressive brain disorder that damages and eventually destroys brain cells, leading to memory loss and changes in thinking and other brain functions. It usually develops slowly and gradually gets worse as brain function declines and brain cells eventually wither and die. Ultimately, AD is fatal, and currently, there is no cure (Alzheimer's Association, 2016). 
The defining characteristics of Alzheimer's Disease Dementia include cognitive deficits in each of the following four criteria (features are individualized): 1) thinking / memory loss; 2) remembering / newly learned material lost; 3) reasoning / problem solving / judgement; 4) and behavioral abilities / mood changes (to the extent that it interferes with daily life and activities). Microscopic brain changes occur long before psychological changes present. Since AD encompasses an entire continuum from the initial pathological changes in the brain that occur before symptoms appear throughout the course of dementia caused by the accumulation of brain changes, it is important to note that those diagnosed with $\mathrm{AD}$ must have symptoms associated with dementia. Research suggests that the brain changes associated with AD may begin 20 or more years before symptoms appear (van Hartan, et al., 2011). When the initial changes occur, the brain compensates for them, enabling individuals to continue to function normally. The typical gradual onset and nature of symptom development often makes diagnosis difficult, often mistaken as normal age-related changes.

As neuronal damage increases, the brain can no longer compensate for the changes and individuals show subtle cognitive decline. Later, neuronal damage is so significant that individuals show obvious cognitive decline, including symptoms such as memory loss or confusion as to time or place. Later still, basic bodily functions such as swallowing are impaired, often contributing to the ultimate decline of oral intake, thus, leading to death due to inanition or other organ compromise. Normal, healthy brains contain roughly 100 billion neurons that form long connections amongst themselves. At such connections, called synapses, information flows in tiny bursts of chemicals that are 
released by one neuron and detected by a receiving neuron (Alzheimer's Association, 2016).

The brain contains about 100 trillion synapses as well. They allow signals to travel rapidly through the brain's neuronal circuits, creating the cellular basis of memories, thoughts, sensations, emotions, movements and skills (Dubois et al., 2013). The accumulation of the protein fragment beta-amyloid (called beta-amyloid plaques) outside neurons and the accumulation of an abnormal form of the protein tau (called tau tangles) inside neurons are two of several brain changes associated with AD (Ariga, Miyatake, \& Yu, 2010). Beta-amyloid plaques are believed to contribute to cell death by interfering with neuron-to-neuron communication at synapses, while tau tangles block the transport of nutrients and other essential molecules inside the neurons. The brains of people with advanced $\mathrm{AD}$ show inflammation, dramatic shrinkage from cell loss, and widespread debris from dead and dying neurons (Fagan et al., 2007). Due to these microscopic changes, characteristics and symptoms of AD manifest. Due to the variability in the area of the brain that is affected, presentation of symptoms also varies on an individual level, making caregiving even more challenging for this population.

\section{Education of staff in LTC}

Presentation of classic features of $\mathrm{AD}$ include a progressive memory loss, a decline in language, cognition, ability to perform activities of daily living (ADLs), personality and behavioral changes, as well as judgment dysfunction (Castellani, Rolston \& Smith, 2010). The variation in clinical presentation poses several challenges for direct and non-direct care providers working specifically within MCUs, as well as on standardization of educational tools to assist in providing the best care possible with a 
PCC approach. Demographically speaking, the vast array of living arrangements for older adults, specifically those with $\mathrm{AD}$ or related dementias, further demands that healthcare providers, consumers and researchers are equipped to care for the individual diagnosed with this provisionally incurable disease. AD can affect people in different ways, with the most common initial symptom being a gradually worsening ability to remember new information. This loss occurs because the first neurons to malfunction and die are usually neurons in the brain regions that are involved in forming new memories. As neurons in other parts of the brain malfunction and die, individuals often experience other difficulties as well (Alzheimer's Association, 2014).

$\mathrm{AD}$ and related dementias inhibit the person's ability to communicate and make their most basic of human needs known, particularly as the disease progresses. Care of people with $\mathrm{AD}$ can involve compensating for their diminishing ability to fulfill basic needs by providing assistance in areas such as activities of daily living (ADLs), mobility, safety and function, toileting, mouth care, and grooming (Edvardsson et al., 2008). PCC focuses on the physical aspects that are required to sustain life, as well as the humanistic qualities that we all possess, irrespective of memory impairment. The basic human needs of a person with $\mathrm{AD}$ or related dementias also include psychosocial aspects, such as feeling safe, having a sense of belonging and acceptance, maintaining social contacts, and feeling respected while experiencing a sense of contribution (Edvardsson et al., 2008).

Of all Medicare beneficiaries age 65 and older with $\mathrm{AD}$ and other dementias, 31 percent live in a nursing home, and 64 percent of those have $\mathrm{AD}$ and other dementias (Bynum et al., 2011). The complexities associated with caring for residents in LTC with a diagnosis of $\mathrm{AD}$ or a related dementia require an entire staff that is trained and well- 
versed on identification of not only the resident as a person, but on those who are also able to identify changes in the residents behavior or personality as it pertains to their care and overall well-being. In 2011, the Centers for Disease Control (CDC) published a report specifying advancements that have been made in the past 50 years relating to disease research and prevention. Despite the notable innovations in medicine and scientific research, AD was noted as still requiring significant attention (Thompson, 2013).

Care of residents, specifically those residing in LTC with a diagnosis of AD or related dementias requires a staff that is adaptable and capable of working in stressful conditions. For the AD or related dementia population, Special Care Units (SCUs) or MCUs have been developed. These units represent a dedicated space within a nursing home or LTC facility that has tailored services for individuals with AD and related dementias. Nursing homes had 75,876 beds in Alzheimer's special care units in 2013, a decrease of 4 percent compared with the previous year (American Health Care Association, 2013). These Alzheimer's special care unit beds accounted for 72 percent of all special care unit beds and 4.5 percent of all nursing home beds.

In LTC facilities, Certified Nurse Assistants (CNAs) comprise the largest segment of staff that works with cognitively impaired residents (McCabe et al., 2007). The majority of CNAs are female, and an increasing number of those are come from diverse ethnic or racial backgrounds (Kramer \& Smith, 2000). Very few would question the fact that direct-care workers have difficult jobs. Turnover rates are high among direct-care workers, and recruitment and retention are persistent challenges (IOM, 2008). 
Additionally, these care workers may not receive the training necessary to provide dementia care.

In a survey of 114 CNAs from five different nursing homes (Blair \& Glaister, 2005), they suggested the following interventions to optimize in-service education: (1) ensure that the educational material is relevant to clinical experience; (2) allow time for hands-on demonstrations and feedback; (3) design programs that meet the needs of adult learners; (4) provide access to information; and (5) allocate time within the clinical day and provide financial support for in-service education.

To assist with the educational training deficit, specific regulations pertaining to LTC facilities in the state of Missouri, and certification through Centers for Medicare and Medicaid Services (CMS) have been established. The Missouri Revised Statutes Chapter 660, Department of Social Services Section 660.05 amendment to include those with AD or related dementias was required to be implemented by January 1, 2002.

According to Missouri regulations: The division shall, by January 1, 2002, establish minimum dementia-specific training requirements for employees involved in the delivery of care to persons with $\mathrm{AD}$ or related dementias who are employed by skilled nursing facilities, intermediate care facilities, residential care facilities, agencies providing in-home care services authorized by the division of aging, adult day-care programs, independent contractors providing direct care to persons with $\mathrm{AD}$ or related dementias and the division of aging. Such training shall be incorporated into new employee orientation and ongoing in-service curricula for all employees involved in the care of persons with dementia. Training shall include at a minimum, the following: 
(1) For employees providing direct care to persons with AD or related dementias, the training shall include an overview of $\mathrm{AD}$ and related dementias, communicating with persons with dementia, behavior management, promoting independence in activities of daily living, and understanding and dealing with family issues;

(2) For other employees who do not provide direct care for, but may have daily contact with persons with $\mathrm{AD}$ or related dementias, the training shall include an overview of dementias and communicating with persons with dementia.

The impetus for the establishment of dementia-specific training throughout the state of Missouri further supports the need for alternative training modalities, as the regulations pertains to direct and non-direct care providers alike. Although the regulation was established in 2002, staff training remains a much-needed area of research and development in order to improve the quality of care, and provision of PCC to residents with a diagnosis of $\mathrm{AD}$ or related dementias.

\section{Positive Physical and Psychosocial influence of online training modules on Person- Centered Care in LTC}

In 2008, The Institute of Medicine (IOM) published Retooling for an aging

America: Building the Health Care Workforce, which identified the need for new models of care for older adults. The article also noted that there will be significant workforce shortfalls in the coming years, but increasing the numbers of geriatric-trained workers will not be sufficient, as it will do nothing to fix the deficiencies in the way care is delivered to older adults or to address the inefficiencies in the current system. Current provisions of care require significant reform in order to improve the care of this 
population. The care that is currently provided to older adults often falls short of acceptable levels of quality. Providers' performance in the delivery of recommended care to older adults varies greatly (RAND, 2004), and there is limited consistent coordination of care among providers.

Staff training, according to the literature, should be designed with consideration to the organizational context while promoting cognitive, affective, and behavioral outcomes (Kraiger, Ford, \& Salas, 1993). In 2008, The Institute of Medicine (IOM) charged the Committee on the Future Health Care Workforce for Older Americans with determining health care needs of Americans older than age 65. Implications of those needs were identified, and the committee proposed that focus must be placed on enhancing the geriatric competence of the entire health care workforce (Institute of Medicine, 2008). Compelling evidence exists for developing educational content, tools, and strategies for teaching specialized skills for a diverse audience of geriatric caregivers (Kowlowitz, Davenport, \& Palmer, 2009). Additionally, studies have made the link between training in dementia care and staff outcomes, such as absenteeism, staff satisfaction, and turnover (Grant, Pothoff, \& Olson, 2001; Maas, Buckwalter, Swanson, \& Mobily, 1994). In other studies, training in dementia care had positive outcomes for both staff and residents (Burgio et al., 2002; Wells, Dawson, Sidani, Craig, \& Pringle, 2000).

Adult participants who have grown accustomed to a visually interactive world or may have had impoverished educational experiences, often lack confidence with traditional, more passive approaches to learning (Bova \& Kroth, 2001; Cohen et al., 2001). Adult learners prefer training they regard as relevant to their lives (Merriam, 2001). Training for caregivers of individuals with AD and related dementias is 
undergoing a fundamental shift from the traditional focus on institutional needs toward PCC, which can improve both caregiver satisfaction and quality of care. Building on the premise that learning should be continual and recognizing that the learning process is an important intermediate step to producing quality care outcomes in a complex care situation (Kemeny, Boettcher, DeShon, \& Stevens, 2006); this proposed study offers a practical method using CARES online training to provide continually accessible information, in an up-to-date and cost-effective fashion. The CARES online format allows staff to stay current on the multiple approaches and examples of providing quality dementia care using actual patients with $\mathrm{AD}$ and actual direct and non-direct care providers. The CARES online format also allows frequent retrieval of information and recurring exposure on an individual basis. Using the CARES online training, staff will have the chance to respond to situations, make choices, watch real-life video scenarios, and become actively involved in the material.

Other potential benefits of the CARES online training format include the opportunity for self-paced learning in a portable format. Requirements for participation and completion of the CARES online training modules include computer accessibility, with an understanding of the English language. Additionally, although training manuals and similar tools are available to assist direct care staff in delivering dementia care, the overall effectiveness of such training approaches remains unclear (Hobday et al, 2010). The time required to modernize hard training manuals, coupled with the time and resources required to hold one-time, face-to-face meetings is not always practical for those employed in LTC. The combination of training, monitoring, and immediate feedback offered with the online CARES format can ensure implementation and 
maintenance of the trained skills necessary to care for residents with AD or related dementias (Bourgeois et al., 2004).

Human interaction is a necessity for residents in LTC facilities. Daily encounters and connections with direct and non-direct care providers who spend the majority of their time with the residents, in particular, have a profound impact on the residents' quality of life (McGilton et al., 2012). Many articles focus primarily on the role that the Registered Nurse (RN) plays in the provision of PCC to residents with dementia, however, attention must also be paid to those non-direct care providers who too interact with the residents in an intimate manner, and are often aware of changes in the residents appearance or behavior before clinical symptoms may appear. Kitwood (1997) supported this notion, stressing, "There is meaning in all behavior, even if it is difficult to interpret." Positive relationships between residents and staff is at the heart of best practice, therefore, addressing the needs of residents who live and die in LTC facilities, and the needs of staff that work there is of equal importance (McCormick et al., 2012).

The opportunities and challenges in providing PCC for people with dementia changes as the condition becomes more severe, but the aim remains, which is to provide a good quality of life for those inflicted with the disease (Bate, 2013). In order to do this, recognition of the essential humanity of all people, regardless of the degree of impairment or illness they experience must be addressed (Downs et al., 2006). The predominant philosophy of care for people with dementia in recent years has been on PCC (Kitwood, 1997). Brooker (2007) has suggested the acronym VIPS to encapsulate the broader meaning of PCC: specifically related to people with dementia. They must be Valued; they must be treated as Individuals; the Perspective of the person with dementia 
must inform our understanding; and the person's Social environment must be attended to because relationships are fundamentally important to sustaining personhood.

The inclusion of the VIPS acronym into the delivery of care for residents inside LTC facilities, specifically those with $\mathrm{AD}$ or related dementias requires a culture renovation. The CARES dementia training modules for staff (to include direct and nondirect care), along with the CARES Observational Tool (COT) represent a potential option for facilities interested in a uniform and systematic process of delivering care that focuses on PCC, as well as a method to evaluate staff on consistent delivery of such care.

LTC facilities can benefit from using direct- and non-direct-care staff efficiently and effectively to improve quality of care and relationships. Integrating new staff into the facility and redesigning work schedules to use all staff effectively require administrative and clinical teamwork. Initiatives to increase staffing should include administrator and clinical staff training on how to creatively modify staff deployment to achieve best practices (Hyer et al., 2009). By providing all direct and non-direct care staff within the MCU the proper educational tools supported and endorsed by the Alzheimer's Association in an online format, the structure of the MCU will, hopefully, change in favor of more PCC, which will ultimately affect resident outcomes.

\section{Donabedian's Structure, Process and Outcomes (SPO) Framework}

Because the MCU represents a unique environment for staff and residents with AD or related dementias alike, this study employed Donabedian's SPO Framework as a guide to the study's rationale and the interpretation of the data. The framework's ability to measure outcomes and link them to actions was important for this particular research study to enhance improvement efforts across direct and non-direct care providers (Polit \& 
Beck, 2008). The subjective nature of defining quality, coupled with the fact that quality of care in nursing homes specifically, has been defined both as an input measure and as an outcome (Kruzich et al., 1992), has made uniformity a complex concept. In 2008, IOM published Retooling for an aging America: Building the Health Care Workforce, which identified the need for new models of care for older adults. On par with this 2008 report, the framework that supports this mission and this particular study is Donabedian's Quality-of-Care.

In the 1960's, Avedis Donabedian established a framework for understanding and evaluating quality improvement work in health care that helped lay the foundation for recognizing that health care quality is measurable and that quality assessment depends on looking at three key components known as the Donabedian Quality Triad (Donabedian, 1966, 2005). Donabedian's SPO framework provides a tool that efficiently articulates three sub-dimensions representing three key components of the healthcare supply chain (Donabedian, 1987). Education is one piece of a complex puzzle, that when placed together with an organization and staff that are ready for change, can potentially improve resident outcomes.

In many cases, the three components of the SPO model have been used as a taxonomy to identify and classify those factors or building blocks that must be put together to assess nursing care performance (Dubois et al., 2013). For this particular research study, the overall triad will refer to direct and non-direct care providers, respectively. The sub-dimension of Structure represents the MCU inside a 120-Bed Private Pay facility outside of Saint Louis, Missouri. Research has shown that the structure of the MCU can have a significant impact on the resident's quality of life, and 
also impact the work that is, and can be performed by the direct and non-direct care staff. Additionally, how staff are trained (or not trained) can have a huge impact on the quality of care provided to residents in LTC, as well as impact their intention to remain in their current positions. Continuing education is a proven means of influencing the knowledge and attitudes of long-term care personnel, and should be a first step toward improving the quality of care in facilities (Almquist, Stein, Weiner, \& Linn, 1981).

Donabedian's theory requires that identified outcomes be clearly linked with the process that caused the outcome. To accomplish this linking, research conducted must help to define the process and justify the causal links with the selected outcomes. Additionally, the identification of desirable outcomes requires dialogue between the participants of care and the direct and non-direct providers of care, which will be evaluated using the COT (Donabedian, 2005).

The figure below was developed from descriptions in the literature to better illustrate the model utilized in the study, and how it is applicable to LTC. 

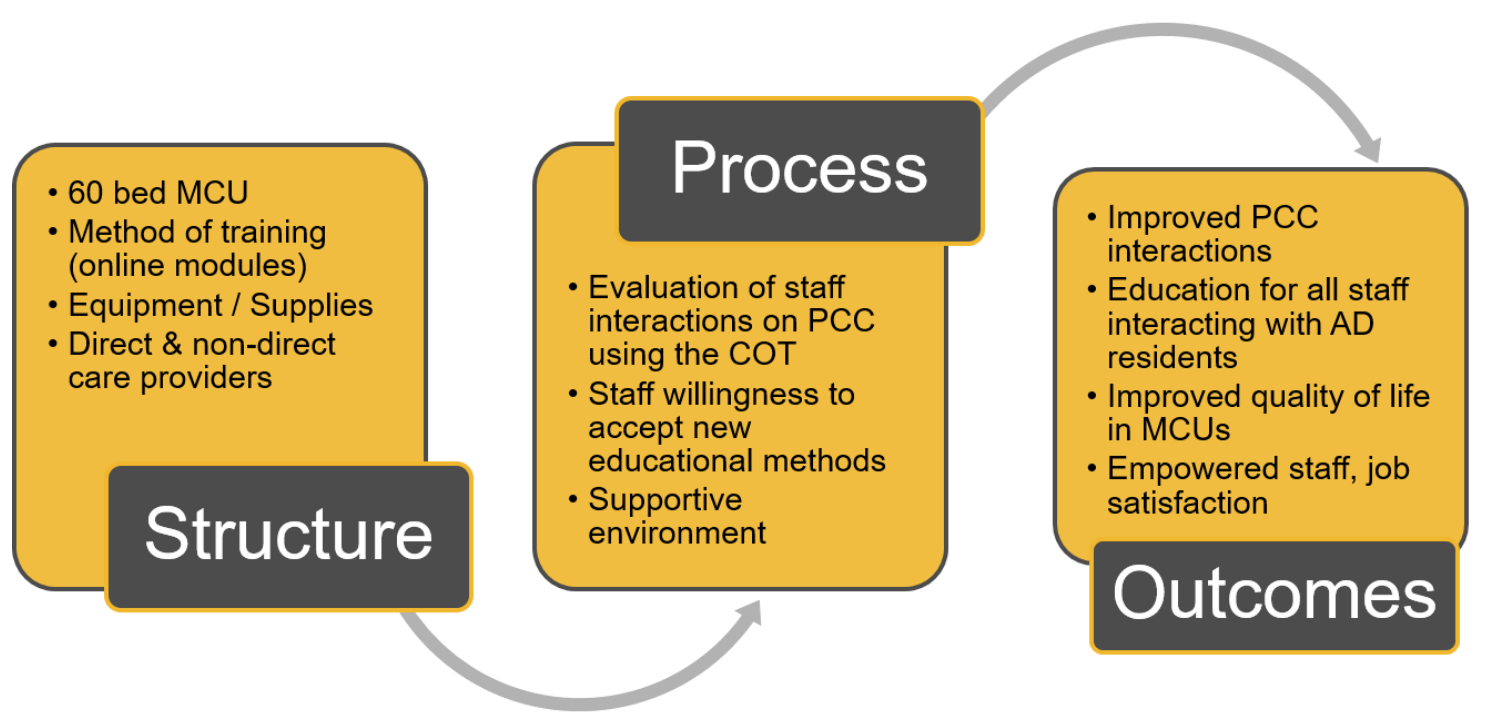

The current study of direct and non-direct workers inside a MCU sought to identify potential areas for increased education and knowledge of caring for residents with a diagnosis of AD or related dementias, with significant association of positive PCC interactions. The staff must be willing and ready to accept the increased educational opportunities, supported in their environment, inclusive of all staff that interact with the residents inside the MCU, and on-board for providing quality care to improve PCC as well as their overall job satisfaction.

Donabedian (1985) believed that evaluation of care could be conceptualized into three dimensions: structure, process and outcomes. He viewed this model as flexible enough to apply to many situations in healthcare, with a direct positive correlation between improvements in the structure of care and improvements in clinical processes that should ultimately, improve patient outcomes (Moore, Lavoie, Bourgeois,\& Lapointe, 2015). This study aimed to achieve this goal by improving staff knowledge and 
improving PCC interactions between staff and residents with AD or related dementias. Although recent research focuses on early dementia diagnosis to improve patient outcomes, few studies have been conducted to include direct and non-direct care providers inside the MCUs of LTC facilities, and the link between increased educational opportunities across the board for overall increased PCC opportunities, and thus, improved quality of life. No studies were found that included both direct and non-direct care providers and that identified online dementia training universally as a variable of interest. This constitutes a gap in the available literature. 


\section{Chapter III}

\section{Design and Methods}

This quantitative, single-group study utilized instrumentation to gather information from direct and non-direct care workers to quantify the impact of CARES dementia training modules completed by all staff on the provision of Person Centered Care (PCC) within a Memory Care Unit (MCU). This chapter describes the study, including sample selection, and design. Data analysis will be described in Chapter IV.

\section{Research Question}

There are two research questions for this proposed study. The questions are as follows:

1. During the three survey periods using the Dementia Knowledge Test (DKT), does knowledge of providing dementia care to residents with $\mathrm{AD}$ or related dementias increase among staff, as measured by the DKT?

2. During the three survey periods, does provision of Person Centered Care (PCC) increase after exposure to the CARES online dementia training modules among staff, as measured by the CARES Observation Tool (COT)?

\section{Hypothesis}

1. Staff exposed to the Dementia Knowledge Test will have higher DKT knowledge scores over the course of the three survey periods.

2. Staff exposed to the CARES online dementia training modules will have increased Person-Centered Care (PCC) interactions over the course of the three survey periods. 


\section{Sample}

A convenience sample of direct and non-direct care providers $(N=26)$ were initially recruited to include all direct and non-direct care providers working within the MCU. Since all staff were invited to participate (the absolute limit of who could participate), a power analysis was not conducted. The investigator personally contacted the Private Pay facility because it advertised itself as being "An expert in dementia care," with a dedicated 60-bed MCU. It should be noted that factors associated with private pay facilities (cost) could be confounding. Based on findings from a previous pilot study conducted by the investigator, it was necessary to include all full-time staff working inside the MCU, versus focusing only on the ones that provide direct care. As long as the work in LTC is seen as tasks and remains focused on clinical skills, rather than on person-centered care and quality of life for the resident, one can easily reduce the requirements for expertise from Registered Nurse (RN) to Licensed Practical Nurse (LPN) or even Certified Nursing Assistants (CNAs), thereby decreasing the cost of care (McGilton, Heath, Bostrom, Mueller, Boscart, McKenzie-Green, Moghabghab \& Bowers, 2012).

Recruitment was completed through initial contact with the two owners of the private-pay facility, as well as the Director of Nursing (DON). After a letter of intent was signed, eligible participants were recruited from the MCU, respectively. Potentially eligible participants attended a presentation, followed by a question and answer session. Two different educational sessions were conducted, to include all staff during their respective shifts. A statement was verbalized aloud to the staff that informed of their right to refuse to participate without any negative consequences, as well as the possibility of 
being requested to participate in any follow-up research. Participation with respect to employment was delineated in the consent form as well. Eligible participants were: a) English as a first language speaking; b) assigned to work in the MCU; c) classified as either direct or non-direct care provider within the MCU; d) employed as full-time status; e) capable of reading at a sixth grade level (as evidenced by signing the consent form) f) able to operate basic functions on a computer (clicking a mouse and typing in a text box). Exclusion criteria included staff that did not speak English as their primary language, staff that did not work with $\mathrm{AD}$ or demented residents, and those that were not employed full-time. It is recognized that staff in the excluded populations may offer unique information that would add to the current body of knowledge, however, inclusion of them was outside of the scope of this small study. It is appreciated that addressing the gaps in the literature related to male employees, education and resident interactions in these populations may be promising areas for future research projects that could build upon this study.

\section{Procedures}

Recruitment of the sample. Twenty-six full-time direct and non-direct care providers cover various roles and responsibilities within the MCU, housed in a 120-bed Private Pay Care Center in the St. Louis, Missouri area were asked to participate in this study. Direct and non-direct care providers were necessary for evaluation, as each brings an important opportunity for providing PCC to the residents with AD inside the MCU. Additionally, statistics support the fact that direct care workers alone will not be able to sustain the existing vacancies, thus, impacting the quality of care that is provided to the residents. According the American Health Care Association 2013 Staff Vacancy \& 
Turnover Survey, "Over $66 \%$ of long-term care employees are nurses or nurse aides. Long-term care services are labor-intensive, so the quality of care depends largely on the performance of the care-giving personnel.

The breakdown of all of the participating staff included: Nine CNAs; three Certified Medication Technicians (CMTs); three LPNs; two RNs, three housekeepers; and six Activity Therapists. Direct care providers were considered to be CNAs, LPNs, and the RNs, while the non-direct care providers included the CMTs, housekeepers, and the Activity Therapists, respectively. Turnover and attrition was anticipated, however, with respect to time, participants were not replaced.

Each eligible employee was presented with an informed consent and HIPAA confidentiality form approved by the University of Missouri-Columbia Internal Review Board. A private room was available for staff choosing to sign consent at the time of recruitment, or staff were allowed to take the consent home for further review. Information on how to contact the PI directly was listed within the consent form, as well as available from the nurse manager of the MCU. MCU and LTC staff were not responsible for any screening or consenting procedures. The investigator collected information from the site weekly in-person; a method agreed upon by the participating LTC facility.

Upon receipt of the signed consent form, a copy was also given to the participating staff. During the first contact with the participant, the investigator determined the potential participant's eligibility. If they chose to participate, time was allowed to answer any questions or concerns that the participant may have about the study process. The study was conducted during the participant's working schedule, 
thereby minimizing barriers to participation for any staff that may need to otherwise make additional trips to complete the study or meet with the researcher. The informed consent was written at a $6^{\text {th }}$ grade reading level, therefore, staff of varying educational levels could participate.

Baseline measurement. After eligibility was determined and consents obtained, the baseline measurement was conducted face-to-face on-site. The PI gathered initial demographic information to include job title, level of education, and length of employment at the respective facility. During the initial interview, the PI also administered the initial Dementia Knowledge Test (DKT) and collected it immediately upon completion. The baseline measurement also included individualized introduction to the CARES online dementia training modules, in which, the participant established their own login and password that was not shared with the PI, and participant was prohibited from sharing their personal login and password information with other staff. Individual DKT and PCC results, as well as completion of CARES online dementia training were not seen by anyone other than the PI.

Retention of participants. In an effort to increase retention, the investigator informed the participants that upon completion of the 10 online dementia training modules, they would receive a CARES certificate that was good for two years, showing advanced dementia training. Additionally, the participants who completed all ten dementia training modules, as well as the evaluation of PCC utilized by the PI, the participants would also be eligible to receive access to the Alzheimer Association's essentiALZ certification course for free. The certification tests and recognizes knowledge of the evidence-based dementia care practices promoted in the Alzheimer's Association 
Dementia Care Practice Recommendations. All Alzheimer's Association essentiALZ and essentALZ Plus certifications are valid for 2 years.

As previously mentioned, attrition was expected. This study originally consented 26 interested participants, and was open to all direct and non-direct care providers. Throughout the course of the study period, four participants voluntarily withdrew consent due to seeking employment elsewhere. Five participants were terminated for various reasons that were not disclosed to the PI. Thus, $(N=17)$ completed the entire study. There are a myriad of reasons for staff to leave the LTC workforce, or choose to not work directly with residents with a diagnosis of $\mathrm{AD}$ or related dementias. According to the IOM: 2008, "Long-term care nurses and CNAs face accident and injury rates exceeding those in the construction and mining industries. Additionally, roughly $30 \%$ of CNAs live at or below the poverty line, while $75 \%$ of CNAs have no employer-sponsored pension." Those statistics, coupled with the fact that the estimated population with diagnosis of AD is projected to reach 7.7 million by 2030 (American Health Care Association, 2013) indicate that all staff working inside LTC facilities are going to need increased education and attention to aid in this looming staffing crisis.

\section{Instrumentation}

Demographics. General demographic information was collected at the baseline interview, including job title, level of education, and length of employment at the respective facility. Participants were asked if they were comfortable completing basic functions on a computer, to include clicking a mouse, or typing in a text box. The online dementia training modules also offered audio descriptions of each screen, so participants had the option to read at their own pace, or listen to audio for each respective module. 
Initial educational information for the MCU staff included a presentation of the study purpose, as well as a demonstration of the $\mathrm{CARES}^{\circledR}$ online dementia training modules, in a train-the-trainer format.

Each participant was provided individual test time inside the Nurse Educator's office for completion of both the DKT, as well as each individual online training module. The private office provided a quiet physical space, as well as uninterrupted time during the participants shift to utilize the online training modules in a format that was most conducive to their learning preference. The maximum time allotted for each individual staff to complete the online training modules (one module per week) was one hour, with a minimum requirement of thirty-minutes (per CMS recommendation). Equipment necessary for online module completion included purchase of the training package on the part of the investigator (to include unlimited user-site license good for one year), a wireless laptop provided and purchased by the investigator, a computer mouse and one headset (optional for participants). Test packets consisting of IRB, HIPAA forms, and the DKT were given to each participant on an individual basis to complete one-on-one (individually) with study investigator and collected immediately post completion by the study investigator. All DKT and COT observations were conducted and collected solely by the PI of this study.

CARES online Dementia Training Modules. This study used the CARES ${ }^{\circledR}$ Dementia Basics and Advanced Care training modules for each participant. CARES stands for (Connect with the resident; Assess behavior; Respond appropriately; Evaluate what works, Share with the team). The training consisted of 10-modules (10 hour), which was a combination of 4 modules from CARES $^{\circledR}$ Dementia Basics ${ }^{\mathrm{TM}}$ and 6 modules from 
CARES $^{\circledast}$ Dementia Advanced Care ${ }^{\mathrm{TM}}$. Participants completed one module per week during their respective shifts, over a ten-week period. The entire training provided core dementia care training - focusing on person-centered care, the changes that happen to thinking skills as dementia progresses, how those changes impact behavior, and how to understand behavior as communication. It also covers specific care topics like making a connection, eating, pain, wandering, falls, and eliminating restraints. The modules included a wide variety of exercises and video clips of real people with dementia, family members, Certified Nursing Assistants (CNAs), and other staff, and is one of only three programs nationwide recommended by Centers for Medicare and Medicaid Services (CMS).

The ten modules completed by each staff member included: Meet Clara Jones, introduction to dementia, understanding behavior as communication, the CARES ${ }^{\circledR}$ approach, making a connection with the person, eating well, recognizing pain, minimizing falls, rethinking wandering and minimizing and eliminating restraints. Upon completion of all ten modules, the participants had the option to complete the Alzheimer's Association essentiALZ Certification Program on their own time, and with accessibility from their own personal computer. The certification tests and recognizes knowledge of the evidence-based dementia care practices promoted in the Alzheimer's Association Dementia Care Practice Recommendations. Completion of the essentiALZ certification program was not a requirement of this study, due to time constraints on behalf of the investigator as well as staff.

Dementia Knowledge Test (DKT). A fifteen question multiple-choice test of direct and non-direct care staffs dementia care knowledge was administered prior to 
completing the ten training modules, immediately after completion of the training modules, and then one month post completion of the training modules. The dementia care knowledge test was developed internally by HealthCare Interactive (HCI) Incorporated and reviewed and modified by staff from the national office of the Alzheimer's Association. The DKT included 15 multiple-choice items. Total time for completion was subjective based on each individual participant, with the majority-completing test in less than ten minutes. Upon initial creation of the dementia care knowledge test, it showed good reliability at pretest (Cronbach's alpha coefficient $=0.94)$. Permission for use was granted by HCI, respectively. The DKT was hand-graded by the investigator, and answers entered in to an Excel Spreadsheet created in collaboration with statistician from the University of Missouri-Columbia. Since the same DKT was administered to the staff at the three designated time points, participants were not allowed to see what test items were missed (if applicable). The DKT mean scores served as data for the three time points.

\section{CARES Observational Tool (COT). The CARES Observational Tool (COT)} pinpoints critical domains of person-centered dementia care interactions. The COT shows evidence of interpersonal connections between the direct and non-direct care worker and the person with dementia, ongoing assessment of behavior on the part of the direct care worker, appropriate responses to the person with dementia's behavior, evaluation of what works by the direct and non-direct care worker, and the sharing of direct and non-direct are workers and the person with dementia are interactions with other staff members (Gaugler, 2013). The COT was completed by the investigator prior to completion of the ten training modules, immediately after completion of training 
modules, and one month post module completion, all in an unobtrusive manner for all residents. The PI had access to participating staff schedules/shifts, and did not announce when COT visits would be occurring so as to not alter the research environment. The COT is comprised of 16 observation items, which were conducted on the part of the investigator unobtrusively during staff shifts. Total score for the observational tool is 16 , with a score of "1" applied for any item observed, and a score of " 0 " if no item was observed during the direct and non-direct staff's interactions with the MCU residents.

Protection of human subjects. The protection of human participants is a requirement of any study utilizing human samples, as well as following Good Clinical Practice (GCP) guidelines. Approval was obtained for this study from the University of Missouri Health Sciences IRB. During participant recruitment (baseline data collection), the PI informed potential participants of the study's purpose and methods, and disseminated the consent form for all potential participants to read invidually, allowing sufficient time to review and ask any questions. Additionally, time was allowed for the potential participants to take the consent form home for further review. Participation was verbalized as voluntary, could be stopped at any time, and would not affect their current employment status. The potential participants were also informed at the inception of the study that the researcher is a nurse, mandated to report elder abuse.

Upon successful recruitment, participants were to choose their own login and password, known only to them. Participants were assigned a unique numeric identifier known only to the PI for review of online module completion. A data key linking the numeric ID to contact information was maintained by the PI in a secured location separate from the research facility. The data key, consent forms, DKT, COT and online 
CARES access containing any information regarding study participants were maintained separately in locked file cabinets within the PI's locked personal office. The PI maintained the only key to the file cabinets. Upon completion of the study, all contact information and the data key were shredded, with the exception of any documentation required by the IRB for retention purposes.

Although it was not anticipated that any harm would come to study participants, participants may have experienced physical or psychological stress from sitting at the computer screen, completing a multiple-choice test during work hours, or from the presence of the PI when completing the COT (although the tool was used unobtrusively). Due to the nature of the online training modules, staff could start and stop at their own pace, with data being saved for future retrieval. All possible methods of maintain participant privacy and safety were utilized. The voluntary nature of study participation was iterated during the informed consent process, and all study procedures took place inside the safe and private environment of the nurse manager's office. Participants were never contacted outside of work. 


\section{Chapter IV}

\section{Results}

This chapter is divided in to two sections: 1) Demographic characteristics of the Memory Care Unit (MCU) environment to include sample, and 2) Findings from the hypothesis testing for the independent variable of CARES online dementia training modules related to the dependent variables of scores from the Dementia Knowledge Test (DKT), as well as the CARES Observational Tool (COT) scores on the provision of Person-Centered Care (PCC). A description of these instruments can be found in Chapter 3.

\section{Demographics}

All participants ( $n=26$ ) were females (by chance) employed within the MCU as full-time direct or non-direct care providers. The sample $(n=26)$ represented the entire staff population inside the MCU, therefore, a power analysis was not conducted. At time of consent, zero participants were determined to not meet eligibility criteria requirements. Four participants voluntarily withdrew consent, verbalizing desire to transfer out of the MCU. An additional 5 were terminated by the study site; however, rationale for termination was not shared with the study investigator for privacy purposes. This left 17 participants who completed the study at pre, post and post-post time points for both sets of data collection. The breakdown of staff consisted of (5) Certified Nurse Assistants (CNAs), (3) Certified Medication Technicians (CMTs), (2) Licensed Practical Nurses (LPNs), (1) Registered Nurse (RN), (2) Housekeepers, and (4) Activity Therapists, all of which were full-time (FT) $(n=17)$. The dependent variables of scores on the Dementia 
Knowledge Test (DKT), as well as the CARES Observational Tool (COT) were measured by mean scores. Distribution of ethnicity, educational level and duration of employment is available in Table 1.

Table 1

Characteristics of Sample at Baseline and Follow-up Data Collection, N(\% )

\begin{tabular}{|l|c|c|}
\hline & $\begin{array}{c}\text { Enrolled } \\
\text { (Baseline; } n=26)\end{array}$ & $\begin{array}{c}\text { Completed } \\
\text { (pre, post \& post-post; } \\
N=17)\end{array}$ \\
\hline $\begin{array}{l}\text { Furrently Employed: } \\
\text { Ethnicity: }\end{array}$ & $26(100 \%)$ & $17(100 \%)$ \\
Caucasian & $10(38.4 \%)$ & $5(29.4 \%)$ \\
African American & $12(46.1 \%)$ & $9(52.9 \%$ \\
Hispanic & $4(15.3 \%)$ & $3(17.6 \%)$ \\
\hline Educational level: & $26(100 \%)$ & $17(100 \%)$ \\
HS Degree or GED & $17(65.3 \%)$ & $10(58.8 \%)$ \\
Some College & $6(23.0 \%)$ & $5(29.4 \%)$ \\
Courses & & $2(11.7 \%)$ \\
Trade School/Comm. & $3(11.5 \%)$ & \\
College Grad & & $1(5.8 \%)$ \\
College Grad + & $2(7.6 \%)$ & $3(17.6 \%)$ \\
\hline Duration of & $4(15.3 \%)$ & $11(64.7 \%)$ \\
employment: & $15(57.6 \%)$ & $2(11.7 \%)$ \\
6 mos or less & $5(19.2 \%)$ & \\
12 mos or less & & \\
1-5 years & & \\
5-7 years & & \\
\hline
\end{tabular}

Friedman's test (non-parametric repeated measures) (ANOVA) was utilized to test the null hypothesis of an intervention effect across three time points. For the DKT, the distribution of scores were different across time $(p=0.0002)$. Summary statistics for the DKT test scores for the pre-tests were lower than for the post and post-posttests (using both the mean and median score). Utilizing a post-hoc Wilcoxon Signed Rank test to evaluate for differences between these dependent test scores, it was concluded that DKT pre-tests were significantly different from the post and post-posttests $(p=0.0001$, 
and $p=0.0008$ ) respectively, but the post and post-posttests were not significantly different from each other $(p=0.3125)$. Results are described in Table 2. Using the number of correct answers out of 15 DKT questions, results indicated that Friedman's test is significant ( $\mathrm{p}<0.0001$ ). Multiple comparison procedures, adjusting for multiple testing, show that pre is significantly less than post and post-post and also that post was significantly less than post-post (adjusted $\mathrm{p}<0.0001$ for all three of these). Figure 1 represents a DKT graph of responses over time, with a line representing each subject. The isolated dots for time 1 (pre) represent subjects who only had data at that time. Figure 2 represents a boxplot, with the line connecting the median values for each time, to reflect changes over time.

Table 2

Friedman's test results for outcome DKT with id and time variables using Mixed pairs; significantly different: Adjusted $p<0.05$

\begin{tabular}{|c|c|c|c|c|c|c|c|c|c|}
\hline Obs & Time & -time & Estimate & StdErr & DF & tValue & Probt & Adjustment & Adjp \\
\hline 1 & Post & $\begin{array}{c}\text { Post- } \\
\text { post }\end{array}$ & -0.5294 & 0.1071 & 32 & -4.94 & $<.0001$ & Tukey & $\begin{array}{c}< \\
.0001\end{array}$ \\
\hline 2 & Post & Pre & 1.2353 & 0.1071 & 32 & 11.54 & $<.0001$ & Tukey & $<$ \\
3 & $\begin{array}{c}\text { Post- } \\
\text { post }\end{array}$ & Pre & 1.7647 & 0.1071 & 32 & 16.48 & $<.0001$ & Tukey & $\begin{array}{c}< \\
.0001\end{array}$ \\
\hline
\end{tabular}


Figure 1

DKT values for subjects over time

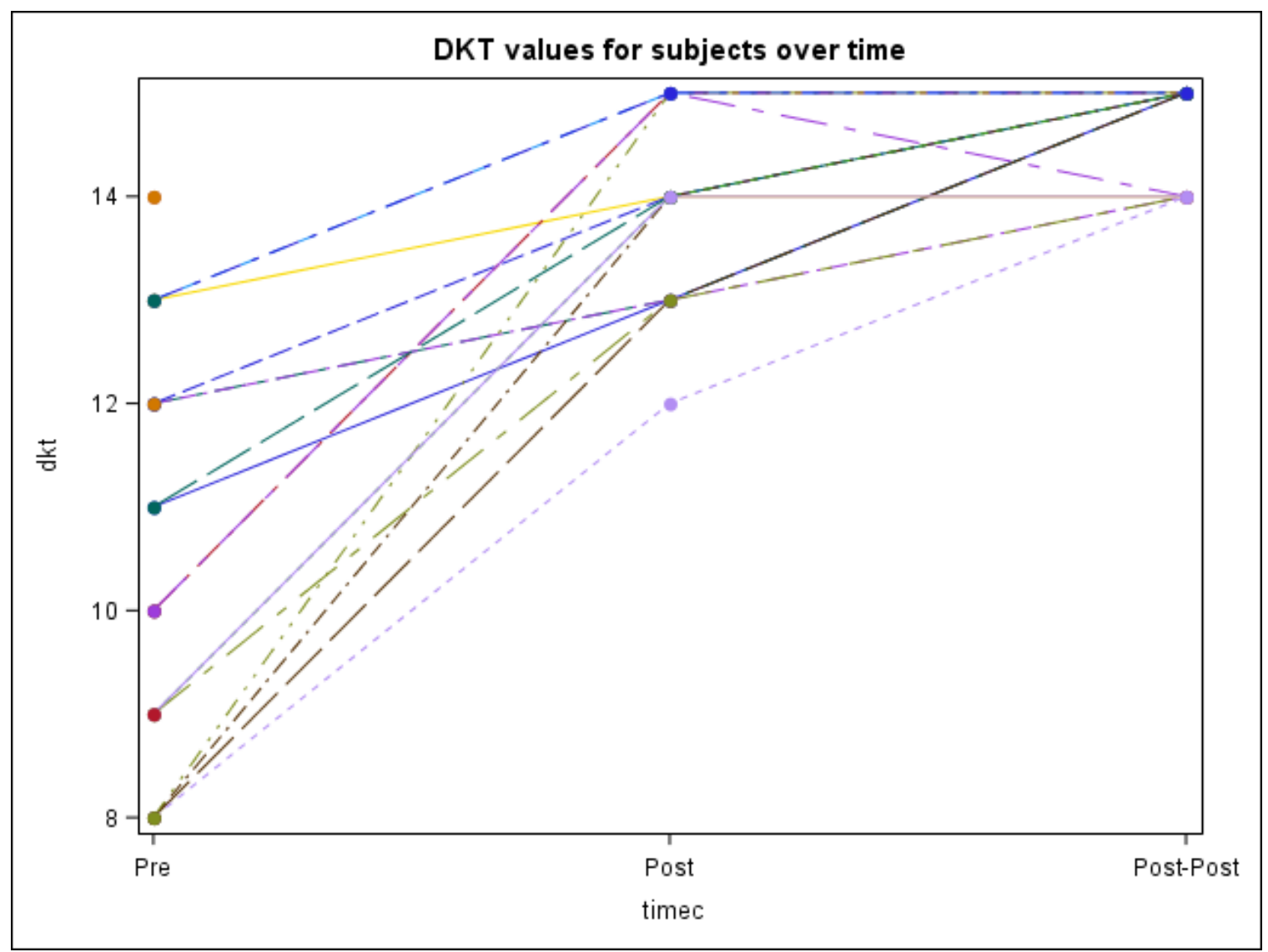


Figure 2

Box-Plots of DKT values over time (Complete data); Medians connected

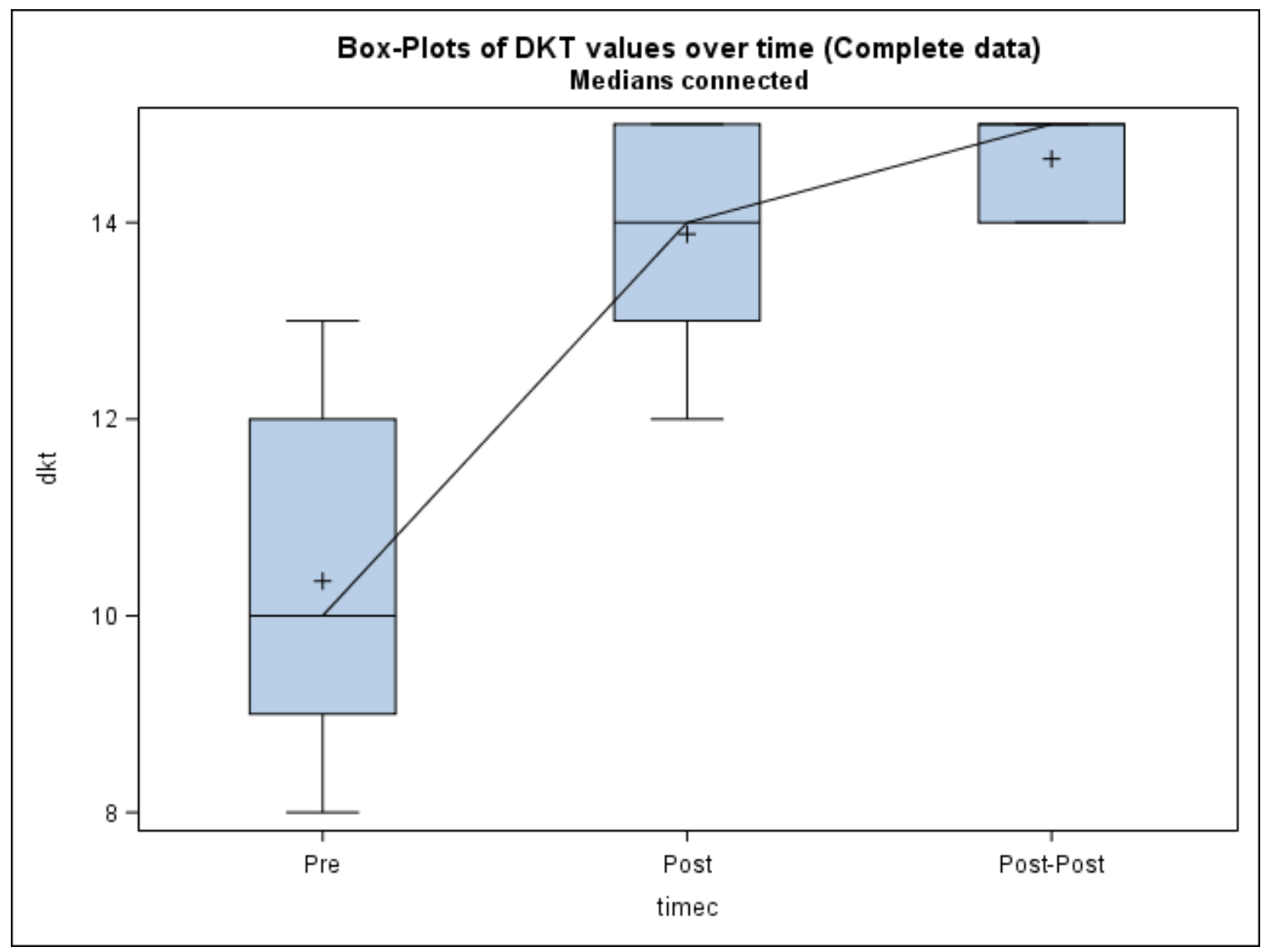

For the CARES Observational Tool (COT), Friedman's test indicated that the distribution of the scores were different across time $(p=0.0001)$. Review of the summary statistics for COT indicated that the test scores for pre-tests were lower than for the post or post-posttests (using both mean and median scores). Again, using a post-hoc Wilcoxon Sign Rank test to evaluate for differences between these dependent test scores, it was concluded that the COT pre-tests were significantly different from the post and post-posttests $(\mathrm{p}=0.0019)$, and $\mathrm{p}=0.0001)$ respectively, as well as the post and postposttests were significantly different from each other $(\mathrm{p}=0.005)$. From the median scores for each COT, it appears that improvement continued to the post-posttests, while for the 
DKT, improvement was isolated to the post-test only. COT results are represented in

Table 3. Similarly, to the DKT data, Figures 3 and 4 represent COT values for subjects over time, as well as a box-plot of COT values over time.

Table 3

Friedman results for outcome COT with id and time variables using mixed pairs significantly different: Adjusted $p<0.05$

\begin{tabular}{|c|c|c|c|c|c|c|c|c|c|}
\hline Obs & Time & time & Estimate & StdErr & DF & tValue & Probt & Adjustment & Adjp \\
\hline 1 & Post & $\begin{array}{l}\text { Post- } \\
\text { Post }\end{array}$ & -0.9063 & 0.1421 & 30 & -6.38 & $\begin{array}{c}< \\
.0001\end{array}$ & Tukey & $\begin{array}{c}< \\
.0001\end{array}$ \\
\hline 2 & Post & Pre & 0.8125 & 0.1421 & 30 & 5.72 & $\begin{array}{c}< \\
.0001\end{array}$ & Tukey & $\begin{array}{c}<. \\
0001\end{array}$ \\
\hline 3 & $\begin{array}{l}\text { Post- } \\
\text { Post }\end{array}$ & Pre & 1.7187 & 0.1421 & 30 & 12.10 & $\begin{array}{c}< \\
.0001\end{array}$ & Tukey & $\begin{array}{c}<. \\
0001\end{array}$ \\
\hline
\end{tabular}

Figure 3

COT values for subjects over time

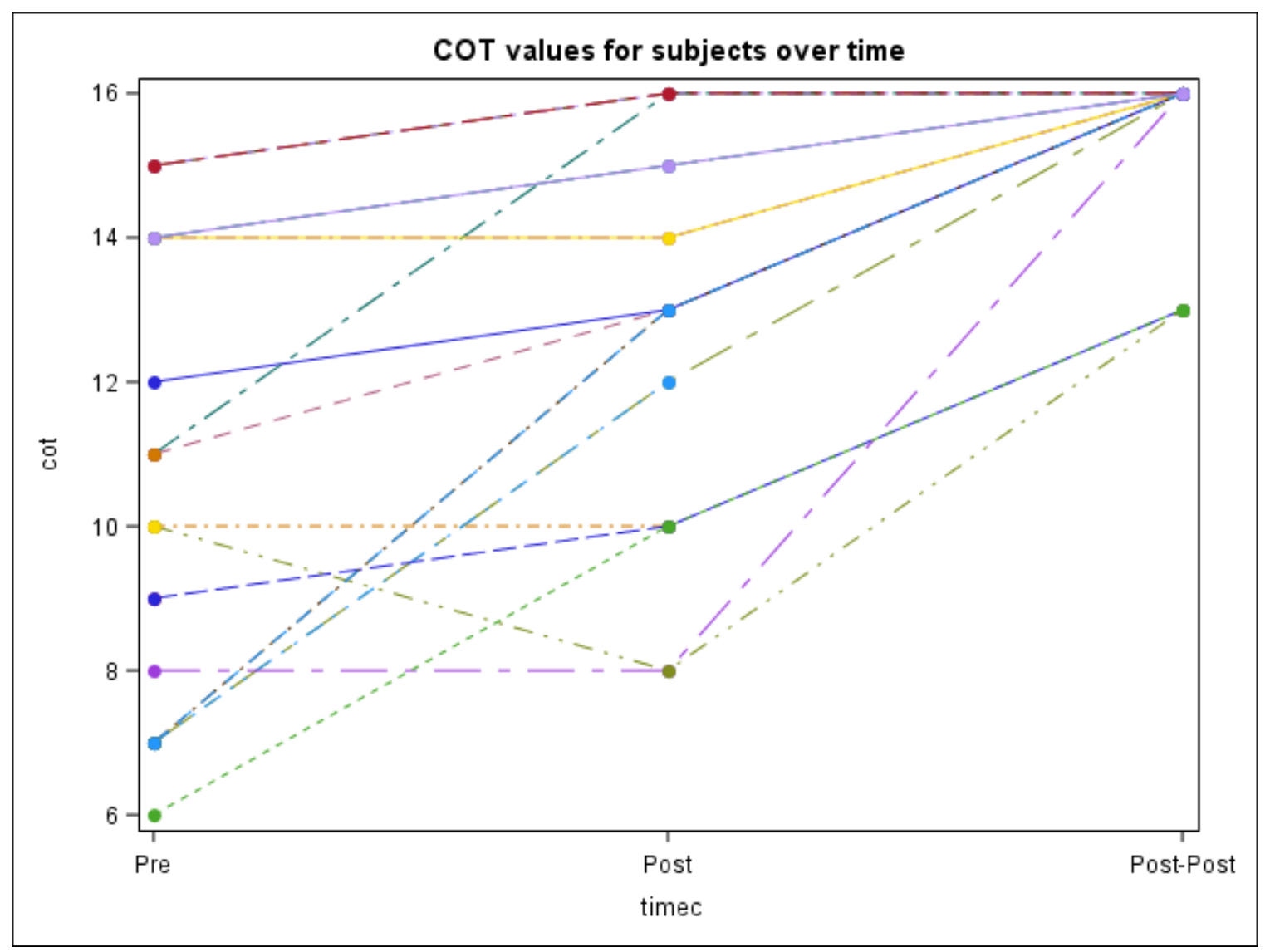


Figure 4

Box Plots of COT values over time (Complete data); Medians connected

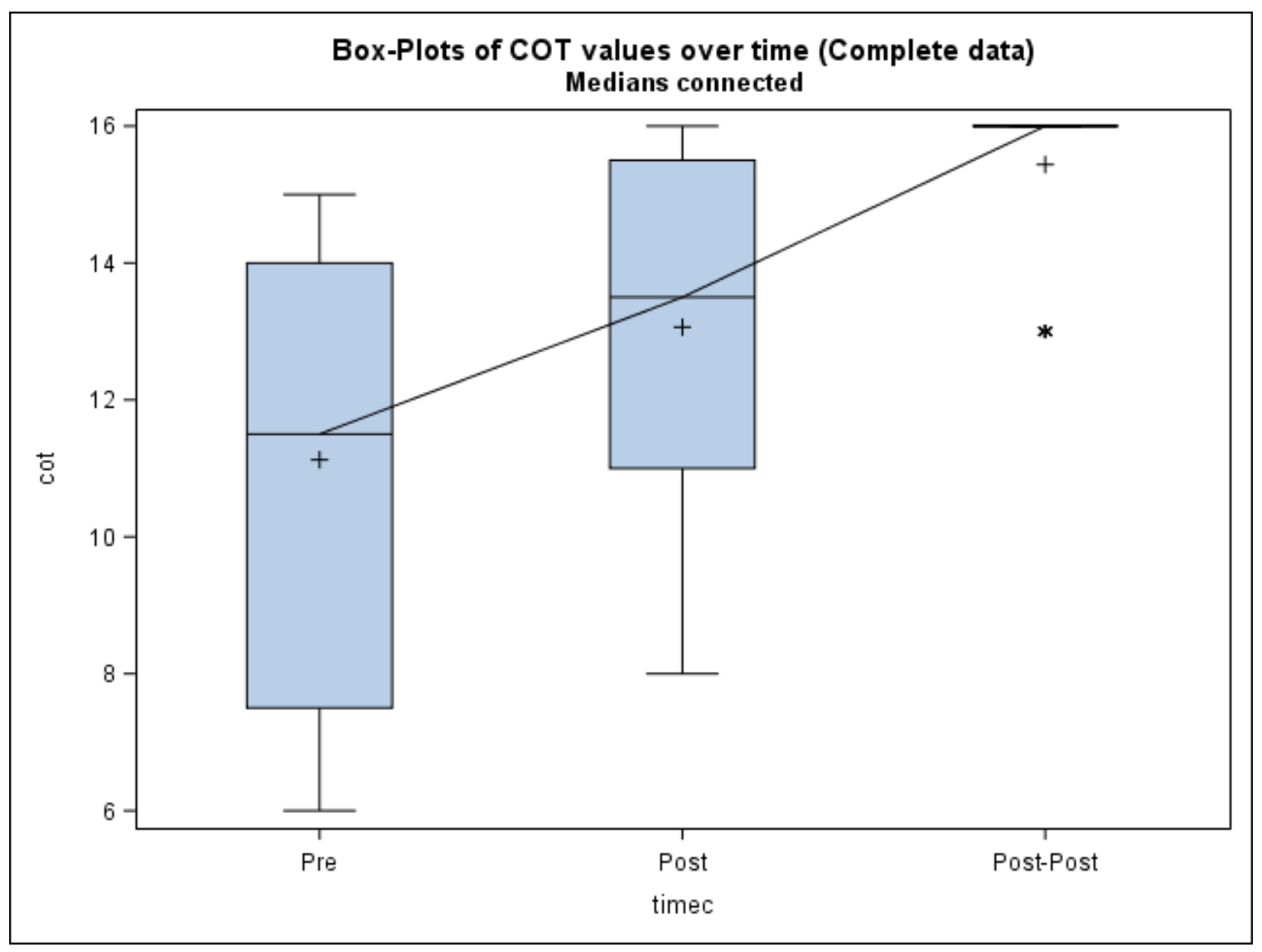

\section{Hypothesis testing}

The level of significance for all hypothesis testing was $\mathrm{p} \leq .05$. The principal hypothesis for this study is 'direct and non-direct care workers inside an MCU who are exposed to the CARES online dementia training modules will have a perceived increase in knowledge of how to best care for residents diagnosed with AD or related dementias,' as measured by the DKT. A secondary hypothesis is 'direct and non-direct care workers who complete the CARES online dementia training modules will deliver increased PCC interactions as measured by the COT with residents diagnosed with $\mathrm{AD}$ or related dementias inside a MCU.' Both hypotheses were supported by statistical analysis. 


\section{Research Questions}

There are two research questions for this proposed study. The questions are as follows:

1. During the three survey periods using the Dementia Knowledge Test (DKT), does knowledge of providing dementia care to residents with $\mathrm{AD}$ or related dementias increase, as measured by the DKT?

2. During the three survey periods, does provision of Person Centered Care (PCC) increase after exposure to the CARES online dementia training modules, as measured by the COT?

The level of significance for all hypothesis testing was $p \leq .05$. For both research questions, Friedman's non-parametric repeated measure was utilized for analysis since multiple measures were obtained from the same subjects. The DKT was initially administered prior to exposure to the CARES online dementia training modules to obtain baseline data on both direct and non-direct care providers. The Pretest yielded statistically significant results $(p<.0001)$ from the Post and Posttests, which was anticipated since there was variability amongst the job responsibilities, length of employment, as well as educational levels between the direct and non-direct care providers. Additionally, the Posttest administered immediately upon completion of all ten CARES online dementia training modules, was significantly less than the Post-Posttest ( $\mathrm{p}<.0001)$. This indicates that knowledge of providing dementia care to residents with $\mathrm{AD}$ or related dementias did, in fact, increase after exposure to the CARES online dementia training modules. The Post and Post-Posttest (which was administered one month after completion of CARES 
dementia training modules), however, were not significantly different from each other ( $p$ $=0.3125)$, indicating that dementia knowledge did not continue over time.

The COT was utilized to support the second research question listed above. Time points for administration of the COT were identical to those of the DKT. The researcher observed all direct and non-direct care providers $(n=17)$ unobtrusively in their natural work environment at the established three time points, for a total of 51 observational experiences. The COT Pretests proved to be statistically different from the Post and Post-Posttest as listed above. Rationales for the indication amongst the differences were again attributed to those mentioned for the DKT. Of importance to note, was that none of the staff or levels of management were informed of when the observations would occur by the researcher, to keep the COT as true as possible. All time points proved to be statistically significant (from the median scores) for each COT, which indicates that the provision of PCC did increase after exposure to the CARES online dementia training modules. 


\section{Chapter V}

\section{Discussion}

The current study measured the efficacy of using online dementia training modules on both direct and non-direct care providers in long-term care (LTC) settings and how this impacted their delivery of Person Centered Care (PCC), as well as their knowledge of caring for residents with a diagnosis of Alzheimer Disease (AD), dementia. This chapter describes the findings of the research in relation to research literature. The sample demographics are discussed relative to previous comparable samples in research, hypotheses results are described, implications for use within the LTC environment are noted for future areas of research, and strengths/limitations of the study are identified.

\section{Demographics}

The analysis of the demographics of the current study note that while the majority of LTC workers are comprised of certified nurse assistants (CNAs), the non-direct care workers employed within the MCU are a cohort of employees with equally important distribution of roles and responsibilities. The sample distribution with $(N=17)$, was almost evenly distributed to include (5) Certified Nurse Assistants (CNAs), (3) Certified Medication Technicians (CMTs), (2) Licensed Practical Nurses (LPNs), (1) Registered Nurse (RN), (2) Housekeepers, and (4) Activity Therapists, all of which were full-time (FT). The CNAs, LPNs and RN comprised the direct care provider cohort, while the CMTs, housekeepers and activity therapists represented the non-direct care providers. Additionally, as previously, mentioned, all participants were women. Since the education sessions were conducted online, and during participants working schedule with the ability 
to start and re-start the training where the participant left off, eliminated a potential barrier of time-consuming training for this particular study.

With the current study, there were no demographic variables that were representative of an association with dementia knowledge pre, post and post-post introduction of the CARES online dementia training modules, nor confounding variables between PCC scores across the same three time points. Several demographic characteristics that have traditionally been associated with decreased knowledge and lower levels of PCC, were not noted to be associated with this study sample, such as poor attendance at traditional in-service sessions (Resnick, Cayo, Galik \& Pretzer-Aboff, 2009), the nursing home environment (administrative support), lack of support for principles of adult learning (Kaasalainen, 2002), and environmental distractions. In many previous studies, the most common method of establishing program effectiveness was testing the nursing assistants before and after the educational intervention, which was the case for this research study. Based on this paper-and-pencil testing, utilized in this study by the DKT, there was evidence that nursing assistants learned new information. Among the studies that included pre- and post-testing of the nursing assistants, only five reported on the rates of attendance at classes (Resnick et al., 2009), which was not an issue with this particular study format.

Increased education and PCC interactions were noted to be significant at different time points within the sample of this study. These findings were not found to be frequently evaluated together in past demographic characteristic associations, as most past research focused solely on education of direct care providers, or on integration of PCC in to the LTC setting. One study by Coogle, Head, Parham \& Zeman, 2004) did 
support that staff training using a person-centered approach to care has been shown to increase knowledge and understanding of persons with Alzheimer's disease, enhance perceived competence and satisfaction in caring for persons with dementia in assisted living facilities (Zimmerman et al., 2005), as well as improve staff behavior in the care of persons with dementia in nursing homes (Boettchner, Kemeny, Deshon, \& Stevens, 2004). The small number of research studies recently completed within the U.S. or specifically inside a MCU makes it difficult to draw appropriate conclusions as to how the current study demographic findings would compare to the general U.S. population. More recent research is needed in both the U.S. to include direct and non-direct care providers inside LTC facilities, and specifically, within MCUs.

\section{Alzheimer's disease and education of staff in MCUs}

The diagnosis of $\mathrm{AD}$ already places the residents in a population at risk, however, for this particular study, the study sample was also noted to be at risk for lack of knowledge of dealing with the cognitive changes that occur over the course of an AD diagnosis. "During the course of the disease, up to $90 \%$ of patients will exhibit behavioral and psychological symptoms of dementia (BPSD), which can include depression, apathy, disinhibition, delusions, hallucinations, aggression, irritability, agitation, anxiety, wandering, and sleep or appetite changes" (Coggins, 2015).

Belonging to a cohort of staff that receive minimal to little dementia-specific training decreases staff satisfaction, as well as the quality of care that is provided to residents with a diagnosis of $\mathrm{AD}$ or related dementias. Additionally, most training is focused on education of CNAs, or RNs, respectively. Although there is general agreement that these direct care providers should have advanced care provision skills, the 
dollars spent to recruit, orient, and train qualified workers are consequently unavailable to support resident care (Coogle, Head \& Parham, 2004). Alternative methods for staff training and development must be considered, allowing for education of more than just direct care providers.

Online learning holds special appeal for busy adults working in under-staffed organizations, such as LTC facilities, who want to advance their learning and/or credentials without prolonged interruptions in their work (American Society for Training and Development [ASTD], 2002; MacDonald \& Gabriel, 1998). Through the utilization of CARES online dementia training modules, there is a cost-effective multimedia educational training program or LTC staff to improve dementia care practices (Hodbay, Savik, Smith \& Gaugler, 2010). Around 70\% of people with dementia who live in LTC facilities have severe dementia and display 'significant' clinical and psychiatric symptoms. Around $10 \%$ of these symptoms are caused by the dementia and $90 \%$ are caused by the way people with dementia are cared for and the environment in which they live (Margallo-Lana et al, 2001). While explanations for the variations within the sample of the current study may not be explicitly reported within other research, one potential answer lay in changes made within the Healthy People 2020 program itself. Included in the comprehensive set of Healthy People 2020 objectives were the new topic area of Dementias, including Alzheimer's disease (AD). According to Healthy People 2020, "This milestone in disease prevention and health promotion creates an opportunity to leverage information technology to make Healthy People come alive for all Americans in their communities and workplaces." 
Demographic variations. Additional variation within the sample of the current study may revolve around the unprecedented turnover rates and overall vacancy rate in both direct and non-direct care positions. High rates of turnover and staff vacancies have multi-layered consequences: patient care suffers, cost of constantly replacing workers soars, and worker job dissatisfaction increases (Stone \& Wiener, 2001). The outlook for an adequate supply of workers to care for the aging baby boom generation is dismal. The National Center for Healthcare Workforce (2004) projected that while personal and home health care assistance will be the fourth most rapidly growing occupation in the United States by 2006, the pool of workers to fill the positions will not be sufficient. Again, research that also addresses the shortage or utilization of non-direct care providers is not frequently a key factor that is included in consideration of LTC turnover or resident outcomes.

Among the key factors that affect the pool of long-term care workers is how American culture values long-term care jobs (Stone \& Wiener, 2001). Low wages, unpleasant work environments, budget and labor constraints, limited career paths, little recognition for a job well done, lack of respect as a valued member of the health care team, and poor job image are issues reported in employee satisfaction surveys and exit interviews (U.S. General Accounting Office, 2001).

\section{Hypothesis Findings}

Knowledge - Staff exposed to the Dementia Knowledge Test will have higher DKT knowledge scores over the course of the three survey periods.

Findings supported the rejection of the null hypothesis related to dementia knowledge, meaning that participants who had lower measures of dementia knowledge 
during their baseline dementia testing improved after exposure to CARES online dementia training $(p=<.0001)$.

Person-Centered Care - Staff exposed to the CARES online dementia training modules will have increased Person-Centered Care (PCC) interactions over the course of the three survey periods.

The current study rejected the null hypothesis related to PCC interactions, demonstrating there was a statistically significant difference between the baseline scores of direct and non-direct care providers who increased their PCC interactions with residents with $\mathrm{AD}$ or related dementias. To date, previous research has not combined implementation of CARES online dementia training modules with increased PCC interactions.

\section{Strengths/Limitations}

The current study demonstrates several strengths and limitations. One strength is the demographic nature of the sample. The professional demographic composition of the sample of the current study is unique in that very few studies were found that included direct and non-direct care providers in their research. The majority of the research findings focused primarily on direct care providers, specifically, certified nurse assistants (CNAs). Since the sample of the current study is similar to that of most employed within a MCU, the generalizability of the findings is improved (Polit \& Beck, 2010). Another strength of the sample is the representation of all staff who were currently employed within the MCU specifically, increasing the applicability of the findings to all staff in numerous MCUs universally. Variations between both rural and urban MCUs may have 
comparisons and contrasts in the resources available to staff demographics depending on the size of the MCU that may impact the applicability of this study.

Retention of the sample from baseline data collection through follow-up is another potential strength of this study. Roughly Sixty-five percent of the staff who completed consent and baseline data also completed follow-up study survey data $(\mathrm{N}=26$ at baseline and $\mathrm{N}=17$ at follow-up). While this particular study fell slightly below the "best-practice goal" of $70 \%$ retention within studies involving human subjects interventions (Lyle et al., 2007), the statistical findings were still significant, and consideration of attrition rates within LTC facilities must be considered. An additional strength of the study was the variety of teaching modalities employed to educate the staff, as well in the data collection methods. All staff was offered multiple ways to obtain information on how to better care for residents with a diagnosis of $\mathrm{AD}$ or related dementias, including a traditional paper-and-pencil, multiple choice test, as well as online computerized training modules that allowed for audio/visual, free-text typing and real-life vignettes of direct and non-direct care providers interacting with real people with a diagnosis of $\mathrm{AD}$ or related dementias. Staffs were allowed to complete the training during working hours (on their own time) via a secure computer in a private location. It should be noted that all staff completed all training in this method.

There were also limitations noted within the current study. One study limitation is related to the sample size. Although all staff employed directly within the MCU initially agreed to participate thus negating the need for a power analysis, attrition did occur, thus decreasing sample size. It is possible that some of the findings that were approaching significance across the study would have reached significance and sustained it with a 
larger sample size $(\mathrm{N})$. The sample of the current study was a convenience sample of direct and non-direct care providers all employed full-time specifically within the MCU who volunteered to participate, however, none of the staff in this study were male (by chance). The sample was neither randomly selected nor randomized to categories within the study as participants self-selected if they were going to participate or not. It is possible that opening the study up to all staff within the facility would have different results on dementia baseline measurements, altering the findings of this study.

The facility chosen for this particular research study was selected because they represented themselves as "Experts in Dementia Care," albeit the respective facility is one of three privately owned facilities within the Saint Louis area. It is possible that utilizing all three sites would have increased sample size, provided more robust research findings, and identified that staff at the various sites have access to different resources (or number of resources) that would impact their dementia knowledge, as well as delivery of PCC. The lack of representation from each site could potentially impact the generalizability of the sample. Due to this study's limited time and resources, as well as working in an environment with fluctuating schedules, it did not address issues of staffs who did not speak English. Staffs who speak other languages are likely to have unique issues and encounters with residents with a diagnosis of $\mathrm{AD}$ or related dementias that represent areas for future research opportunities.

\section{Implications for Nursing}

On par with the Institute of Medicine's (IOM) progress report on Future of Nursing 2020 goals, this study was interested in enhancing education (with respect to education of staff in LTC working with demented residents), as well as removing barriers 
to practice and care. According to the committee, "no single profession, working alone, can meet the complex needs of patients and communities." While the IOM focuses primarily on the role of the $\mathrm{RN}$, the overarching goal is universal and particularly applicable to this study, as it includes all staff working within the MCU collaboratively. It is imperative that nurses returning to school focus on the current crisis that exists within education of staff with respect to $\mathrm{AD}$ and dementia care knowledge. A key component in the success of a dementia care program is the role of the Nurse. "Compelling evidence exists for developing educational content, tools, and strategies for teaching specialized skills for a diverse audience of geriatric caregivers. Providing these resources is challenging in light of the shortage of nurses and nurse educators, especially in geriatrics.” (Kowlowitz, Davenport \& Palmer, 2009).

With the rapidly expanding geriatric population, bedside nurses need to be wellinformed concerning the most current evidence-based, theory-guided practice, especially concerning common geriatric syndromes, such as falls, urinary incontinence, and eating difficulties (Mezey \& Zwicker, 2006). Theories of aging help guide gerontological nursing practice. Theories originate from numerous scholarly disciplines (biology, economics, psychology, sociology, etc.). Each theory concerns distinct facets of aging or characteristics of elderly people, but no theory covers everything. No single theory prevails in gerontology (Moberg, 2001).

\section{Implications for Research}

The current study indicates several areas for future research that may prove beneficial for enhancing knowledge and provision of PCC for residents with AD or related dementias residing in MCUs. The primary aim of this study was to evaluate for 
perceived changes in PCC care after completing the CARES dementia training modules, using the COT over a five-month period (January 2015-May 2015), as well as evaluate for changes in dementia care knowledge after completing the CARES dementia training modules, using the CARES Dementia Knowledge Test (DKT). Future research studies should include intervention studies to identify specific interventions that increase the breadth of knowledge provided to all staff employed within LTC facilities. Utilization of the SPO model to serve as a foundation for which facilities could expound upon to initiate universal staff training should be researched to support (or not) the inclusion of this educational method in all LTC facilities.

To more clearly identify potential barriers to education of direct and non-direct care providers working within LTC facilities, a qualitative analysis including private pay facilities, as well as Medicare/Medicaid funded facilities should be conducted. Inclusion of both types of facilities and identification of specific educational methods utilized within both may help develop new interventions or promote the use of the intervention employed within this study as a viable option.

\section{Conclusion}

The number of individuals affected with $\mathrm{AD}$ or related dementias, and the growing economic and societal impact that accompanies the diagnosis is nothing short of devastating. To date, $\mathrm{AD}$ has no cure. Today, there is a worldwide effort under way to find better ways to treat the disease, delay its onset, and prevent it from developing (Alzheimer's Association, 2016). The current study aimed at finding unique and realistic educational modalities for those who care and interact with residents in MCUs who have a diagnosis of $\mathrm{AD}$ or related dementias. As the disease progresses, communication and 
care becomes increasingly more difficult. Given the ambiguity of resident behaviors, indeterminate level of staff education, high attrition rates and complexity of resident needs, it is imperative that facilities implement educational strategies that can encompass all staff where they are. The CARES online dementia training modules and evaluation using of the COT represents one such intervention.

Nursing implications include identification of the increased need for education focused on this vulnerable population, and serving as role models and change agents within LTC facilities. Future research should focus on identification of methods used by LTC facilities to educate their entire staffs that have been successful, identification of barriers specific to staff training, and studies to assess efficacy of interventions developed that follow the SPO model of changing the environment to change the outcome. Improving educational opportunities for all staff in LTC is imperative. These direct and non-direct care providers are in a unique position to impact a vulnerable population, and are often not armed with the resources that they need in order to do so effectively. How many people can leave shift work knowing that their daily interactions can have meaning for a resident who may not be able to remember their own family members face or name? Residents with AD or related dementias deserve better care. Staff working in LTC facilities deserves better training opportunities across the board. The Nursing profession can assist in leading the charge in assisting with $\mathrm{AD}$ and dementia education while we wait for a cure. 


\section{BIBLIOGRAPHY}

Almquist, E., Stein, S., Weiner, A., \& Linn, M.W. (1981). Evaluation of continuing Education for long-term care personnel: Impact upon attitudes and knowledge. Journal of the American Geriatrics Society, 29: 117-122.

Alzheimer's Association (2013). Alzheimer's disease facts and figures. Alzheimer's \& Dementia, 9(2).

Alzheimer's Association (2014). Available at www.alz.org/alzheimers_disease_facts_and figures.asp. Retrieved March 23, 2014

Alzheimer's Association (2015). Available at Www.alz.org/alzheimers_disease_facts_and figures.asp. Retrieved January, 11, 2015.

Alzheimer's Association (2016). Available at www.alz.org/alzheimers_disease_facts_and figures.asp. Retrieved February, 04, 2016

American Health Care Association. (2013). LTC stats: Nursing facility operational Characteristics report, 2012. Available at http://www.ahcancal.org/research data. Retrieved June 02, 2013.

Ariga, T., Miyatake, T., \& Yu, R.K. (2010). Role of proteoglycans and Glycosaminoglycans in the pathogenesis of Alzheimer's disease and related disorders: Amyloidogenesis and therapeutic strategies_A review. Journal of Neuroscience, 88: 2303-2315. 
Bate, H. (2013). Ensuring quality of life for people with severe dementia. Nursing and Residential Care, 15(3): 157-160.

Blair, C.E., \& Glaister, J.A. (2005). Making the most of Nursing Homes' number one resource. Health Care Management Review, 30(2): 110-115.

Boetttcher, I.F., Kemeny, B., Deshon, R.P., \& Stevens, A.B. (2004). A system to Develop staff behaviors for person-centered care. Alzheimer's Care Today, 5(3): 188-196.

Bova, B., \& Kroth, M. (2001). Workplace learning and generation X. Journal of Workplace Learning: Employee Counseling Today, 13(2): 57-65.

Brooker, D. (2007). Person-centred dementia care: Making services better $\left(2^{\text {nd }}\right.$ ed). London, UK: Jessica Kingsley Publishers.

Brownie, S., \& Nancarrow., S. (2012). Effects of person-centered care on residents and staff in aged-care facilities: A systematic review. Clinical Interventions in Aging, 8, 1-10. http://dx.doi.org/10.2147/CIA.S38589

Burgio, L.D., Stevens, A.B., Burgio, K.L., Roth, D.L., Paul, P., \& Gerstle, J. (2002). Teaching and maintaining behavior management skills in the nursing home. The Gerontologist, 42: 487-496.

Bourgeois, M.S., Dijkstra, K., Burgio, L.D., \& Allen, R.S. (2004). Communication Skills training for nursing aides of residents with dementia. Journal of Clinical Gerontologist, 27(1-2).

Bynum, J., Andrews, A., Sharp, S., McCullough, D., \& Wennberg, J.E. (2011). The Care Span: Fewer hospitalizations result when primary care is highly integrated into a continuing care retirement community. Health Affairs (Project Hope), 30(5): 975-984. http://doi.org/10.1377/hlthaff.2010.1102

Castellani, R.J., Rolston, R.K., \& Smith, M.A. (2010). Alzheimer Disease. Disease-amonth, 56(9): 484-546. http://doi.org/10.1016/j.disamonth.2010.06.001 
Centers for Medicare \& Medicaid Services (CMS) 2013b. "Funding Opportunity:

Support for demonstration ombudsman programming services serving Medicare-Medicaid enrollees." http:www.cms.gov/Newsroom/MediaReleaseDatabase/Factsheets/2013Fact-Sheet-Items/2013-06-27.html. Retrieved July 20, 2013.

Chicago Health and Aging Project (CHAPS). (2003). Design of the Chicago health and aging project (CHAP). Journal of Alzheimers Disease, 5(5): 349-55.

Cohen, S.L., Dove, D.W., \& Bachelder, E.L. (2001). Time to treat learners as consumers. Training and Development, 55(1): 54-57.

Coggins, M. (2015). Behavioral expressions in dementia residents. Geriatric Medicine. Retrieved December 12, 2016.

Coogle, C.L., Head, C.A., Parham, I.A., \& Zeman, S. (2004). Person-centered care and the workforce crisis: A statewide professional development initiative.

Educational Gerontology, 30(1): 1-20. https://doi.org/10.1080/03601270490248428

Crandall, L.G., White, D.L., Schuldeis, S., Talerico, K.A. (2007). Initiating person centered care practices in long-term care facilities. Journal of Gerontological Nursing, 33(11): 47-56.

Donabedian, A. (1987). The Quality of Care: How can it be assessed? Journal of American Medical Association, 260: 1743-1748.

Donabedian, A. (2005). Evaluating the Quality of Medical Care. The Milbank Quarterly, 83(4): 691-729. doi:10.1111/j.1468-0009.2005.00397.x

Downs, M. (2006). The emergence of the person in dementia research. Ageing and Society, 17(5): 597-607.

Dubois, C.A., D’Armour, D., Tchouaket, E., Clarke, S., Rivard, M., \& Blais, R. (2013). Associations of patient safety outcomes with models of nursing care organization at unit level in hospitals. International Journal for Quality in Health Care: 1-8. 
https://doi.org/10.1093/intqhe/mzt019

Edvardsson, D., Winblad, B., \& Sandman, P. (2008). Person-centred care of people with severe Alzheimer's disease: Current status and ways forward. Lancet Neurology, 7(4): 362-367.

Fagan, A.M., Roe, C.M., Xiong, C., Mintun, M.A., Morris, J.C., \& Holtzman, D.M. (2007). Cerebrospinal Fluid tau/B-Amyloid42 ratio as a prediction of cognitive decline in nondemented older adults. Archives of Neurology, 64(3): 343-349. doi: 10.1001/archneur.64.3noc60123

Gaugler, J.E., Hobday, J.V., \& Savik, K. (2013). The CARES Observational Tool: A Valid and reliable instrument to assess person centered dementia care. Geriatric Nursing, 34(3), 194-198.

GAO (General Accounting Office). 2001b. Recruitment and retention of nurses and nurse aides is a growing concern. Washington, DC: United States General Accounting Office.

Grant, L., Potthoff, S.J., \& Olson, D. (2001). Staffing and administrative issues in special care units. Alzheimer's Care Quarterly, 2(3): 22-27.

Hobday, J., Savik, K., Smith, S., \& Gaugler, J. (2010). Feasibility of internet training for care staff of residents with dementia: The CARES program. Journal of Gerontological Nursing, 36(4): 13-21. doi: 10.3928/00989134-20100302-01

Hyer, L., Yeager, C.A., Hilton, N., \& Sacks, A. (2009). Group, individual, and staff therapy: An efficient and effective cognitive behavioral therapy in long-term care. American Journal of Alzheimer's Disease and other Dementias, 23(6): 528-539.

Institute of Medicine. (2008). Retooling for an aging America: Building the healthcare workforce. Retrieved January 27, 2010, from the National Academies Press web site: http://www.nap.edu/catalog.php?record_id=12089 
Kaasalainen, S. (2002). Staff development and long-term care of patients with dementia. Journal of Gerontological Nursing, 28(7): 39-46.

Kemeny, B., Boettcher, I., DeShon, R., \& Stevens, A. (2006). Innovations in community-based and long-term care. Using experiential techniques for staff development: Liking, learning, and doing. Journal of Gerontological Nursing, 32(8): 9-14.

Kitwood, T. (1997). Dementia reconsidered: The person comes first. Buckingham, United Kingdom: Open University Press.

Kowlowitz, V., Davenport, C., \& Palmer, M. (2009). Development and dissemination of web-based clinical simulations for continuing geriatric nursing education. Journal of Gerontological Nursing, 35(4): 37-43.

Kraiger, K., Ford, J.K., \& Salas, E. (1993). Application of cognitive skill-based, and Affective theories of learning outcomes to new methods of training evaluation. Journal of Applied Psychology, 78: 311-328.

Kramer, N.A., \& Smith, M.C. (2000). Training nursing assistants to care for nursing home residents with dementia. In V. Molinari (Ed.), Professional psychology in long term care (pp. 227-256). New York: Hatherleigh Press

Kruzich, J.M., Clinton, J.F., \& Kelber, S.T. (1992). Personal and environmental Influences on nursing home satisfaction. The Gerontologist, 32(3): 342-350. https://doi.org/10.1093/geront/32.3.342

Linkage of NCHS Population Health Surveys to Administrative Records from Social Security Administration and Centers for Medicare \& Medicaid Services. U.S. Department of Health and Human Services, Centers for Disease Control and Prevention, National Center for Health Statistics, (2015). Retrieved from: https://books.google.com/books?isbn=0840606737

Lyle, C.M., Kay, L.S., Crepaz, N., Herbst, J.H., Passin, W.F. et al. (2007). Bestevidence interventions: Findings from a systematic review of HIV behavioral 
interventions for US populations at high risk, 2000-2004. American Journal of Public Health, 97: 133-143. https://doi.org/10.2105/AJPH.2005.076182

Maas, M., Buckwater, K.C., Swanson, E. \& Mobily, P.R. (1994). Training key to job satisfaction. Journal of Long Term Care Administration, 22(1): 23-26.

MacDonald, C.J., \& Gabriel, M.A. (1998). Toward a partnership model for Web-Based learning. The Internet and Higher Education, 1(3): 203-216.

https://doi.org/10.1016/S1096-7516(99)80167-5

Margallo-Lana, M., Swann, A., O'Brien, J., et al.: Prevalence and pharmacological Management of behavioral and psychosocial symptoms amongst dementia Sufferers living in care environments. International Journal of Geriatric Psychiatry, 16: 39-44.

McCabe, M.P., Davison, T.E., \& George, K.K. (2007). Effectiveness of staff training programs for behavioral problems among older people with dementia. Aging \& Mental Health, 11(5): 505-519. Doi: 10.1080/13607860601086405

McCormick, B., Roberts, T., Meyers, J., Morgan, D., \& Boscart, V. (2012). Appreciating the "person" in long-term care. International Journal of Older People Nursing, 7: 284-294.

McGilton, K.S., Heath, H., Chu, C.H., Bostrom, A., Mueller, C.M., Boscart, V., \& Bowers, B. (2012). Moving the agenda forward: A person-centered framework in long-term care. International Journal of Older People Nursing, 7(4): 303-309. Doi: 10.1111/opn.12010

Merriam, S.B. (2001). Andragogy and self-directed learning: Pillars of adult learning theory. New Directions for Adult and Continuing Education, 89: 3-13.

Mezey, M., \& Zwicker, D.A. (2006). Urinary Incontinence. Geriatric nursing protocols for best practice ( ${ }^{2 \text { nd }}$ ed., pp. 83-98). New York, NY: Springer.

Moberg, D.O. (2001). Aging and Spirituality: Spiritual dimensions of Aging theory, research, practice, and policy. New York, NY: Routledge. 
Moore, L., Lavoie, A., Bourgeois, G., Lapointe, J. (2015). Donabedian's structureprocess-outcome quality of care model: Validation in an integrated trauma system. Journal Trauma Acute Care Surgery, 78(6): 1168-1175.

Doi: 10.1097/TA.000000000000663

National Center for Health Workforce Analysis. 2004. Nursing aides, home health aides, and related health care occupations-national and local workforce shortages and associated data needs. Rockville, MD: Health Resources and Services Administration.

Nolan, M. (2001). Successful aging: Keeping the "person" in person-centered care. British Journal of Nursing, 10: 450-454.

Polit, D.F., \& Beck, C.T. (2008). Nursing Research: Generating and assessing evidence for nursing practice. ( $8^{\text {th }}$ ed.). New York: Lippincott, Williams \& Wilkins.

Polit, D.F., \& Beck, C.T. (2010). Essentials of Nursing Research: Appraising evidence for nursing practice. $\left(7^{\text {th }}\right.$ ed). New York: Lippincott, Williams \& Wilkins.

Rand Law and Health Resource Brief. (2004). Centers for the Study of Aging. RAND Press.

Rantz, M.J., \& M. Flesner, et al. (2003). Getting the basics right. Care delivery in nursing homes. Journal of Gerontological Nursing, 29(11): 15-25.

Resnick, B., Cayo, J., Galik, E., \& Pretzer-Aboff, I. (2009). Implementation of the 6week educational component in the Res-Care intervention: Process and outcomes. Journal of Continuing Education, 40: 353-360.

Stone, R.I., \& Wiener, J.M. (2001). Who will care for us? Addressing the long-term care workforce crisis. Washington, D.C.: Urban Institute.

Thompson, J. (2013). Rethinking Memory Care. Long-Term Living. pp. 39-41. van Harten, A.C., Kester, M.I., Visser, P.J., Blankenstein, M.A., Pijenburg, Y.A., et al., 2011. Tau and p-tau as CSF biomarkers in dementia: A meta-analysis. Clinical Chemistry Laboratory Medicine, 49: 353-366. 
Wells, D., Dawson, P., Sidani, S., Craig, D., \& Pringle, D. (2000). Effects of an abilities focused program on morning care on residents who have dementia and on caregivers. Journal of the American Geriatrics Society, 48: 442-449.

Zimmerman, S., Sloane, P.D., Williams, C.S., Reed, P.S., et al. (2005). Evaluating the quality of life of long-term residents with dementia. Gerontologist, 45(Special Issue I): 37-49. 
VITA

Erin Cattoor was born June 20, in St. Louis, Missouri. She graduated in 2007 from the University of Missouri-St. Louis with her Master's Degree in Nursing Education. She started her doctoral degree in the fall of 2010, and completed the pursuit May 2018.

Erin has been involved in the nursing profession since 2000, working the majority of her nursing career in Critical Care. She became a nurse educator at a private university in St. Louis in 2006, teaching in traditional, accelerated and weekend-college programs. Erin currently works at a major academic and research institution in the St. Louis area, focusing on Alzheimer Disease Dementia clinical research. 